\title{
ESTIMATIVA DA LÂMINA MÁXIMA DE ESCOAMENTO SUPERFICIAL NA BACIA DO RIO SÃO MIGUEL, MINAS GERAIS
}

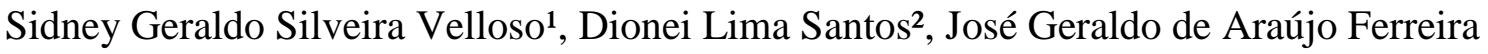 \\ Filho $^{3}$
}

\author{
${ }^{1}$ Mestrando em Ciência Florestal; Departamento de Engenharia Florestal; Universidade Federal de Viçosa; \\ sidney.velloso@hotmail.com \\ 2Dionei Lima Santos; Departamento de Engenharia Agrícola; Universidade Federal de Viçosa; dionei_lima12@hotmail.com \\ 3José Geraldo de Araújo Ferreira Filho; gfilho @ hotmail.com
}

RESUMO: O escoamento superficial ocorre quando a duração da precipitação (de baixa intensidade e longa duração) é superior ao tempo para saturação do solo, ou quando a intensidade de precipitação é superior à taxa de infiltração de água no solo. Por estar relacionado à erosão, o conhecimento de seus parâmetros é importante para mitigação dos efeitos prejudiciais que podem ocorrer. Utilizou-se dois modelos para estimativa da lâmina máxima de escoamento superficial: método do Número da Curva e Balanço de Água na Superfície do Solo. Utilizou-se, para tanto, imagens orbitais provenientes do sensor RapidEye e, dados hidrológicos da área de estudo. Os resultados indicaram, pelos dois métodos, que a presença de vegetação de porte florestal reduz a lâmina máxima de escoamento superficial, seguido por pastagens e terras cultivadas.

Palavras-chave: hidrologia, ciclo hidrológico, uso do solo

\section{ESTIMATING MAXIMUM DEPTH OF SURFACE RUNOFF IN THE SÃO MIGUEL RIVER BASIN, MINAS GERAIS ESTATE}

ABSTRACT: Surface runoff occurs when the duration of precipitation (of low intensity and long duration) is greater than the time needed for soil saturation, or when intensity of rainfall is higher than water infiltration rate in the soil. Because it is related to erosion, the knowledge 
of its parameters is important to mitigate the harmful effects that may occur in this process. It was used two models to estimate the maximum depth of surface runoff: Curve Number method and Water Balance in the Soil Surface. Therefore, it was used orbital images from the sensor RapidEye and, hydrological data from the study site. Results showed, by the two methods, that the presence of forest vegetation reduces the maximum depth of surface runoff, followed by pasture and croplands.

Keywords: hydrology, hydrological cycle, land use

\section{INTRODUÇÃO}

O ciclo hidrológico consiste na transferência de água, entre os compartimentos do meio, numa sequência fechada de fenômenos naturais (QUEIROZ \& OLIVEIRA, 2013). Nos continentes, após iniciado o processo de precipitação, a água proveniente das chuvas pode encontrar três destinos: retenção pela cobertura vegetal, infiltração na matriz edáfica e/ou armazenamento em depressões superficiais, ou escoamento sobre a superfície terrestre.

O escoamento superficial ocorre quando a precipitação prevalece durante um intervalo de tempo superior àquele necessário à saturação do solo ou quando a intensidade de precipitação supera a taxa de infiltração de água no solo (GRIEBELER et al., 2001). Uma vez que este fenômeno está diretamente associado à erosão hídrica (PRUSKI et al., 2001), tornase necessário o conhecimento de parâmetros relacionados ao mesmo, visando tomadas de decisão que venham a mitigar os efeitos prejudiciais causados pelo processo. Vários autores procuraram modelar o processo de escoamento superficial sob diversas circunstâncias naturais (LANGE \& HAENSLER, 2012; MUÑOZ-VILLERS \& MCDONELL, 2012).

Em projetos estruturais para contenção da erosão ou controle de inundações, informações acerca do processo de escoamento superficial são imprescindíveis. Quanto ao objetivo, pode-se armazenar o montante de água gerado no processo, condicionando o indivíduo a conhecer o volume total escoado. No entanto, quando a necessidade for a 
condução do excedente de água, torna-se necessário conhecer a vazão escoada (LOMBARDI NETO et al., 1994).

Pruski et al. (1997) propuseram um modelo (Balanço de Água na Superfície do Solo (BASS)) para estimativa da lâmina total escoada, baseando-se nos princípios físicos inerentes ao processo. Para tanto, consideraram a premissa de precipitação uniforme para a área analisada, bem como solo com umidade próxima à saturação. $\mathrm{O}$ modelo culminou na estimativa da lâmina total de escoamento superficial por meio da equação 1:

$E S_{M}=P T-I_{\alpha}-I-e v$

Onde:

$$
\begin{aligned}
& \mathrm{ES}_{\mathrm{M}}=\text { lâmina total de escoamento superficial }(\mathrm{mm}) ; \\
& \mathrm{PT}=\text { precipitação total }(\mathrm{mm}) ; \\
& \mathrm{I}_{\mathrm{a}}=\text { abstrações iniciais }(\mathrm{mm}) ; \\
& \mathrm{I}=\text { infiltração de água no solo }(\mathrm{mm}) ; \mathrm{e} \\
& \text { ev = evaporação }(\mathrm{mm}) .
\end{aligned}
$$

. Nesse método, considera-se a evapotranspiração como nula Os demais parâmetros da equação 1 podem ser obtidos a partir das equações apresentadas em Griebeler et al. (2001).

Outro modelo (Número da Curva (NC)) utilizado para determinação da lâmina total de escoamento superficial é apresentado pelo Soil Conservation Service (SCS) (RALLISON, 1980), vinculado ao United States Department of Agriculture (USDA). Nesse, utiliza-se a relação, encontrada a partir de informações obtidas pelo SCS-USDA, apresentada na equação 2:

$\frac{\mathrm{I}}{\mathrm{S}}=\frac{\mathrm{ES}}{\mathrm{P}_{\mathrm{e}}}$ 
Onde:

I = infiltração acumulada após o início do escoamento superficial (mm);

$\mathrm{S}=$ infiltração potencial $(\mathrm{mm})$;

$\mathrm{ES}=$ escoamento superficial total $(\mathrm{mm}) ; \mathrm{e}$

$\mathrm{P}_{\mathrm{e}}=$ escoamento potencial $(\mathrm{mm})$.

Nesse modelo, a variável S é obtida a partir do conhecimento do chamado Número da Curva (NC), que utiliza informações sobre o material biofísico de cobertura presente na área.

Diante das informações apresentadas, o objetivo do presente estudo foi obter a lâmina de escoamento superficial para uma bacia hidrográfica no estado de Minas Gerais, a partir dos modelos Balanço de Água na Superfície do Solo e Número da Curva.

\section{MATERIAL E MÉTODOS}

A área de estudo (Figura 1) está localizada no estado de Minas Gerais, sob

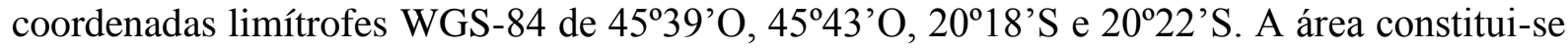
da sub-bacia do Rio São Miguel, localizada no perímetro municipal de Arcos, centro-oeste mineiro. A área da bacia é de aproximadamente $244 \mathrm{~km}^{2}$, sendo composta por aproximadamente $42,72 \%$ de florestas, $48,31 \%$ de pastagens e $8,97 \%$ de terras cultivadas. 


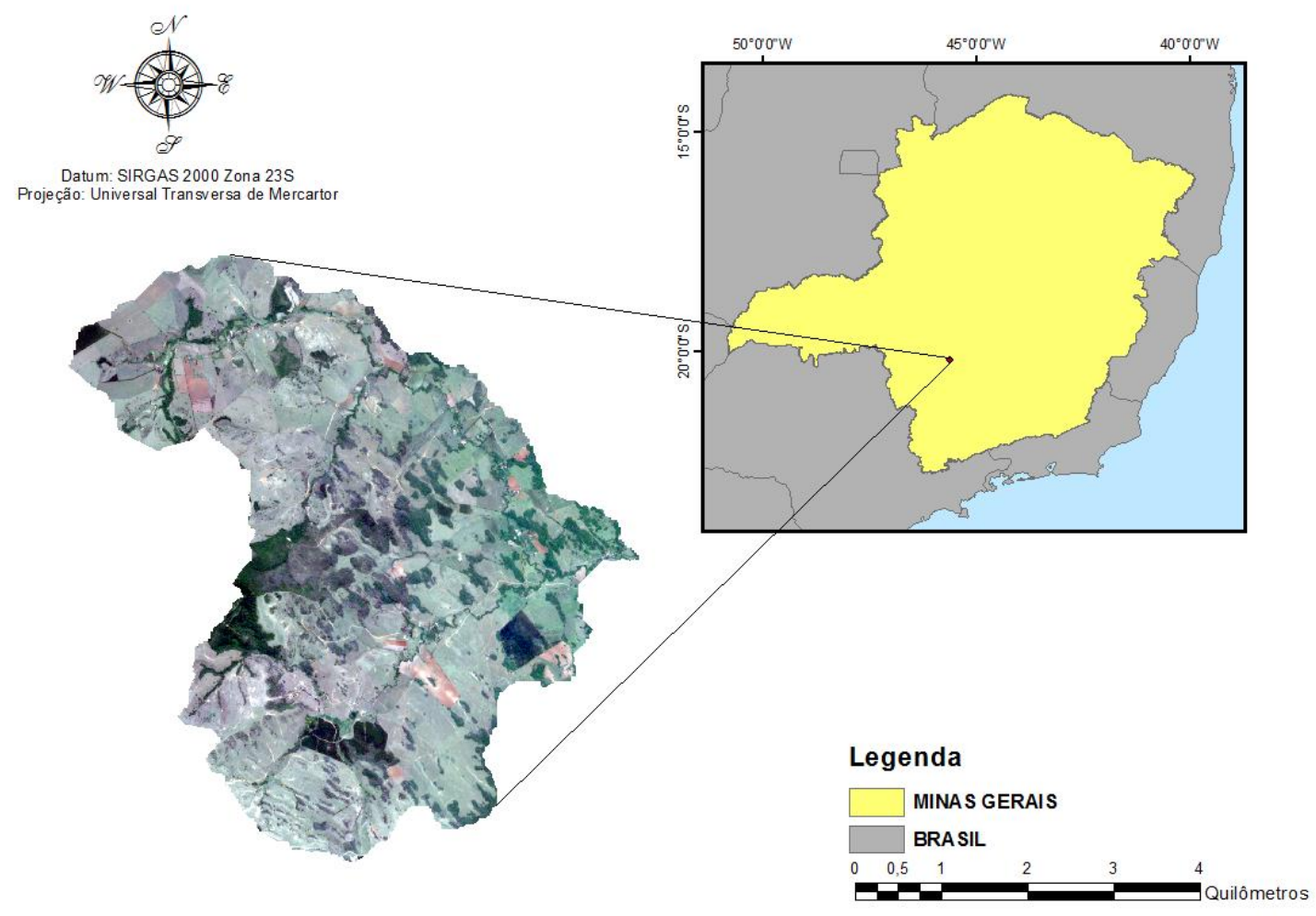

Figura 1. Área da bacia em estudo com destaque para a imagem 3R2G1B Rapideye de 2013.

Figure 1. Study watershed area highlighting the composition 3R2G1B of a Rapideye image dating of 2013.

Foram obtidas duas cenas provenientes do sensor RapidEye, cedidas pelo Ministério do Meio Ambiente (MMA) através do portal www.geocatalogo.mma.gov.br. De posse das cenas RapidEye, foram derivadas informações espaciais da distribuição das classes de cobertura do solo na bacia. Para tanto, foi utilizada a classificação supervisionada por regiões, a partir do algoritmo de classificação Bhattacharya (MATHER, 2004) (Figura 2). 


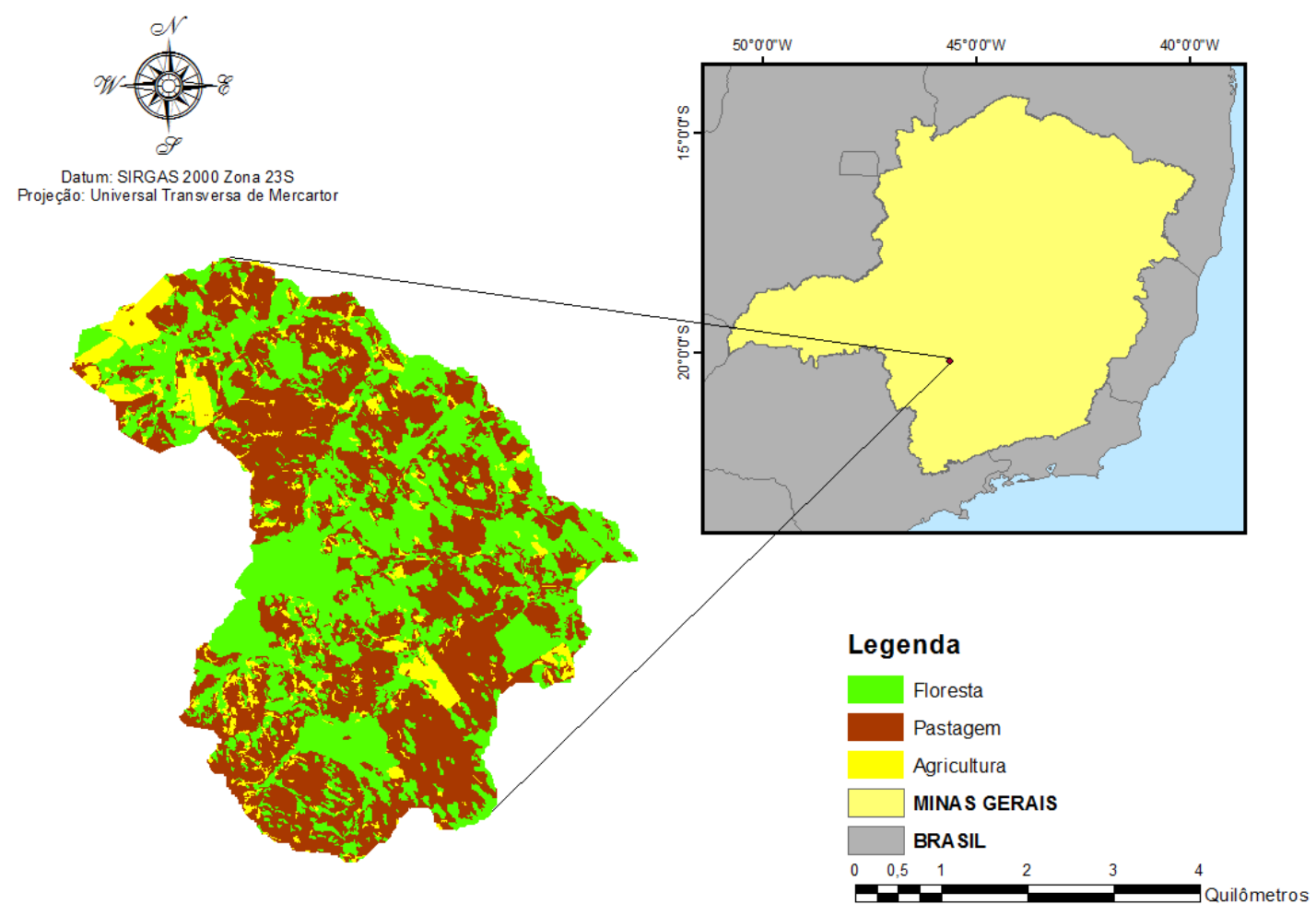

Figura 2. Mapa temático de cobertura do solo da bacia em estudo.

Figure 2. Thematic map of the soil cover from the study watershed.

Para estimativa da lâmina de escoamento superficial foi utilizado o software Excel 2013. Para proceder ao cálculo da lâmina máxima de escoamento superficial pelos métodos utilizados no presente trabalho (BASS e NC) foi necessário obter a equação de intensidade, duração e frequência para o local de estudo. Para tanto, utilizou-se o software Pluvio 2.1, obtendo-se a seguinte equação para o município de Arcos:

$\mathrm{i}=\frac{\mathrm{aT}}{(\mathrm{t}+\mathrm{c})^{\mathrm{d}}}=\frac{826,446 \mathrm{~T}^{0,194}}{(\mathrm{t}+10,188)^{0,742}}$

Onde: 
$\mathrm{i}=$ intensidade máxima de precipitação $\left(\mathrm{mm} \cdot \mathrm{h}^{-1}\right)$;

$\mathrm{T}=$ período de retorno (anos).

$\mathrm{t}=$ duração da chuva $(\min )$;

a,b,c,d = parâmetros empíricos.

Processamentos espaciais necessários à execução do presente estudo foram realizados nos softwares ArcMap 10.2.2 e SPRING 5.2.7.

\section{RESULTADOS E DISCUSSÃO}

Para o método Número da Curva, foram utilizadas três durações de precipitação, sendo os tempos de 6, 12 e 24 horas com período de retorno de 10 anos. A partir dos resultados obtidos pelo mapeamento temático, foram geradas as estimativas para lâmina de escoamento superficial apresentadas na Tabela 1, em função da cobertura e tempo de duração da precipitação.

Tabela 1. Lâmina máxima de escoamento superficial obtida pelo método do número da curva. Table 1. Maximum depth of surface runoff obtained by the curve number method.

\begin{tabular}{cccccccc}
\hline Tempo (h) & Cobertura & CN & $\boldsymbol{S}(\mathbf{m m})$ & $\boldsymbol{I} \boldsymbol{a}(\mathbf{m m})$ & $\begin{array}{c}\text { PT } \\
(\mathbf{m m})\end{array}$ & $\begin{array}{c}\text { ES por } \\
\mathbf{c o b e r t u r a} \\
(\mathbf{m m})\end{array}$ & $\begin{array}{c}\mathbf{E S} \\
(\mathbf{m m})\end{array}$ \\
\hline & Pastagem & 84,4 & 46,9 & 9,4 & 96,3 & 56,4 & \\
6 & Floresta & 78 & 71,6 & 14,3 & 96,3 & 43,7 & 51,87 \\
& Terras Cultivadas & 88,7 & 32,4 & 6,5 & 96,3 & 66 & \\
\hline 12 & Pastagem & 84,4 & 46,9 & 9,4 & 116,3 & 74,3 & 69,12 \\
\hline
\end{tabular}




\begin{tabular}{cccccccc}
\hline & & & & & & & \\
& Floresta & 78 & 71,6 & 14,3 & 116,3 & 59,9 & \\
& Terras Cultivadas & 88,7 & 32,4 & 6,5 & 116,3 & 84,9 & \\
& Pastagem & 84,4 & 46,9 & 9,4 & 139,8 & 95,9 & \\
\multirow{2}{*}{24} & Floresta & 78 & 71,6 & 14,3 & 139,8 & 79,9 & 90,11 \\
& & & & & & & \\
& Terras Cultivadas & 88,7 & 32,4 & 6,5 & 139,8 & 107,3 & \\
\hline
\end{tabular}

Verifica-se coerência nos resultados obtidos para lâmina de escoamento superficial por esse método. Destaca-se que a classe "Floresta" apresentou a menor lâmina de escoamento superficial para todos os tempos de duração da precipitação. Tal resultado vai ao encontro do exposto por Ilstedt (2007) e Tucci \& Clarke (1997) ao afirmarem que coberturas florestais tendem a reter maior parcela da precipitação incidente e promovem maior infiltração de água no solo.

A classe "Terras Cultivadas" apresentou a maior lâmina de escoamento superficial para todos os tempos de duração da precipitação. Pinheiro et al. (2009) ao avaliarem a taxa de infiltração para diferentes culturas, floresta e pastagem, encontraram maiores taxas de infiltração para matas e florestas, intermediárias para cultivos e menores para pastagens. Esse resultado confronta aqueles apresentados no presente estudo, no entanto, deve-se destacar que o método Número da Curva utiliza os valores tabelados para o parâmetro $\mathrm{S}$ advindos de tabelas geradas pelo SCS-USDA. Portanto, são utilizados valores obtidos para condições diferentes daquelas verificadas para o local de estudo.

Chaves \& Piau (2008), ao utilizarem o método Número da Curva para estimativa do volume de escoamento superficial para condições de plantio convencional, plantio direto e cerrado nativo, obtiveram valores médios de 41,1 mm.ano ${ }^{-1}$, 26,5 mm.ano ${ }^{-1}$ e 12 mm.ano-1, respectivamente. Tais resultados mostram a influência de uma cobertura vegetal mais 
estruturada na infiltração de água no solo e, consequentemente, na redução do escoamento superficial, corroborando com os resultados apresentados no presente estudo.

A Tabela 2 apresenta os resultados para lâmina de escoamento superficial obtidos pelo método Balanço de Água na Superfície do Solo.

Tabela 2. Valores obtidos para lâmina máxima de escoamento superficial pelo método do balanço de água na superfície do solo.

Table 2. Values obtained for maximum depth of surface runoff by the method of water balance in the soil surface.

\begin{tabular}{ccccccc} 
Cobertura & $\mathbf{C N}$ & $\mathbf{I a}(\mathbf{m m})$ & $\mathbf{P T}(\mathbf{m m})$ & $\mathbf{I}(\mathbf{m m})$ & $\begin{array}{c}\text { ES por } \\
\text { cobertura } \\
(\mathbf{m m})\end{array}$ & $\mathbf{E S}(\mathbf{m m})$ \\
\hline Pastagem & 84,4 & 9,4 & 40 & 16,41 & 14,32 & \\
Floresta & 78 & 14,3 & 40 & 15,05 & 10,74 & 12,99 \\
Terras Cultivadas & 88,7 & 6,5 & 40 & 17,10 & 16,55 & \\
\hline
\end{tabular}

Assim, como no modelo Número da Curva, a classe "Floresta" foi aquela que apresentou menor volume de escoamento superficial, seguida por "Pastagem" e "Terras Cultivadas". Portanto, fica indicado que a classe florestal atua no sentido de reduzir a lâmina de escoamento superficial. Yuan et al. (2015) apontam que, em regiões onde a declividade seja superior a $5^{\circ}$, áreas de cobertura florestal, arbustiva e/ou gramínea não devem ser convertidas para uso agrícola.

O valor de lâmina de escoamento superficial apresentado pelo BASS é menor que aquele apresentado pelo $\mathrm{NC}$, uma vez que os tempos para duração da precipitação são diferentes. Ao trabalhar com tempo crítico (onde o escoamento superficial é máximo) relacionado à uma taxa de infiltração estável de $40 \mathrm{~mm} \cdot \mathrm{h}^{-1}$, foi encontrado o valor de 27,57 
minutos para duração da precipitação no BASS. Esse valor é bem menor que a duração de 6 horas de precipitação (menor valor de duração apresentado para o NC), o que confirma a afirmativa anterior quanto às divergências entre valores de precipitação relacionadas às durações da precipitação para os métodos.

As vantagens do método BASS são a possibilidade da estimativa de lâmina máxima de escoamento superficial para condições mais próximas à realidade brasileira (uma vez que o método CN utiliza parâmetros derivados de tabelas internacionais) e a eficiência propriamente dita do modelo em estimar esse parâmetro (GRIEBELER et al., 2001; PRUSKI et al., 2001).

\section{CONCLUSÕES}

A cobertura florestal foi aquela que apresentou menor lâmina máxima de escoamento superficial dentre as feições analisadas, indicando assim, que o manejo da cobertura vegetal do solo mitigará efeitos danosos provenientes do processo de escoamento superficial.

\section{REFERÊNCIAS}

CHAVES, H. M. L.; PIAU, L. P. Efeito da variabilidade da precipitação pluvial e do uso e manejo do solo sobre o escoamento superficial e o aporte de sedimento de uma bacia hidrográfica do Distrito Federal. Revista Brasileira de Ciência do Solo, v. 32, n. 1, p. 333343, 2008.

GRIEBELER, N. P.; PRUSKI, F. F.; JÚNIOR, MARTINS JÚNIOR, D.; SILVA, D. D. Avaliação de um modelo para a estimativa da lâmina máxima de escoamento superficial. Revista Brasileira de Ciência do Solo, v. 25, n. 2, p. 411-417, 2001.

ILSTEDT, U.; MALMER, A.; VERBEETEN, E.; MURDIYARSO, D. The effect of afforestation on water infiltration in the tropics: A systematic review and meta-analysis. Forest Ecology and Management, v. 251, n. 1-2, p. 45-51, 2007.

LANGE, J.; HAENSLER, A. Runoff generation following a prolonged dry period. Journal of Hydrology, v. 464-465, n. 1, p. 157-164, 2012. 
LOMBARDI NETO, F.; BELLINAZZI JÚNIOR, R.; LEPSCH, I. F.; OLIVEIRA, J. B.; BERTOLINI, D.; GALETI, P. A.; DRUGOWICH, M. I. Terraceamento agrícola. Campinas: Secretaria da Agricultura e do Abastecimento do Estado de São Paulo, Coordenadoria de Assistência Técnica Integral, 1994. 39p. Boletim Técnico CATI, 206.

MATHER, P. M. Computer Processing of Remotely-Sensed Images. Chichester: John Wiley \&Sons, $3^{\text {a }}$ ed. 2004, 324p.

MUÑOZ-VILLERS, L. E.; MCDONELL, J. J. Runoff generation in a steep, tropical montane cloud forest catchment on permeable volcanic substrate. Water Resource Res., n. 48, v. 9, 2012.

PINHEIRO, A.; TEIXEIRA, L. P.; KAUFMANN, V. Capacidade de infiltração de água em solos sob diferentes usos e práticas de manejo agrícola. Ambiente \& Água, v. 4, n. 2, p. 188 199, 2009.

PRUSKI, F. F.; BRANDÃO, V. S.; SILVA, D. D. Escoamento superficial. Viçosa: UFV, $1^{\text {a }}$ ed. 2003, 88p.

PRUSKI, F. F.; RODRIGUES, L. N.; SILVA, D. D. Modelo hidrológico para estimativa do escoamento superficial em áreas agrícolas. Revista Brasileira de Engenharia Agrícola e Ambiental, v. 5, n. 2, p. 301-307, 2001.

QUEIROZ, A. T.; OLIVEIRA, L. A. Relação entre produção e demanda hídrica na bacia do Rio Uberabinha, estado de Minas Gerais, Brasil. Sociedade \& Natureza, v. 25, n. 1, p. 191204, 2013.

RALLISON, R. E. Origin and evolution of the SCS Runoff equation. In: Symposium on watershed management, New York, 1980. Anais. New York, American Society of Civil Engineers, 1980. p. 912-924.

TUCCI, C. E. M.; CLARKE, R. Y. Impacto das mudanças da cobertura vegetal no escoamento: revisão. Revista Brasileira de Recursos Hídricos, v. 2, n.1, p. 135-152, 1997.

YUAN, Z.; CHU, Y.; SHEN, Y. Simulation of surfasse runoff and sediment yield under different land-use in a Taihang Mountains watershed, North China. Soil \& Tillage Research, v. 153 , n. 1, p. 7-19, 2015 